\title{
DERIVED NUMBERS WITH RESPECT TO FUNCTIONS OF BOUNDED VARIATION*
}

BY

R. L. JEFFERY

1. Introduction. The present paper is a supplement to our previous paper. $\dagger$ It deals with the distribution of values of the derived numbers of a function $F(x)$ with respect to a function of bounded variation $\alpha(x)$, and the possibility of determining $F$ from one of these derived numbers when the derived number in question is finite. That $F$ can be determined from its derivative with respect to $\alpha$ when this derivative is finite has been shown by Lebesgue, $\ddagger$ and also by the present writer. $\S$ The method used by Lebesgue involves a transformation which reduces integration with respect to a function of bounded variation to ordinary integration. That of the present writer is direct. Lebesgue remarks $\|$ concerning his method that it does not seem suitable for handling the corresponding problems which arise when the derivative with respect to $\alpha$ is replaced by derived numbers with respect to $\alpha$. We have found that these problems will yield to the direct method of treatment, but the

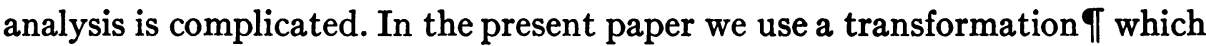
is different from that of Lebesgue, but which, like his, reduces the operations of differentiation and integration with respect to a function of bounded variation to the corresponding operations in the ordinary sense.

We shall be concerned with functions $F$ which are constant on intervals throughout which $\alpha$ is constant, and for which $F(x-0)$ and $F(x+0)$ both exist at the discontinuities of $\alpha$, and consequently at the discontinuities of $\omega$, the variation function of $\alpha$. At the points of discontinuity of $\omega$ let $\psi(x, h)$ $=\{F(x+h)-F(x \mp 0)\} / m \omega(x, h), \mp$ holding according as $h \gtrless 0$. At the points of continuity of $\omega$, let $\psi(x, h)=\{F(x+h)-F(x)\} / m \omega(x, h)$ when $m \omega(x, h) \neq 0$, $\psi(x, h)=0$ when $m \omega(x, h)=0$. Then the upper and lower limits of $\psi(x, h)$ as $h$

* Presented to the Society, April 15, 1933; received by the editors June 11, 1933, and, in revised form, March 19, 1934.

$\dagger$ Non-absolutely convergent integrals with respect to functions of bounded variation, these Transactions, vol. 34, pp. 645-675, the notation of which is carried throughout the present paper. The paper cited is referred to in what follows as T. It contains some typographical errors: p. 656 , line $16, D_{\omega} F$ should read $\left|D_{\omega} F\right|$; the numerator of the last inequality on this page should read $\mid F\left(x_{k+1}-0\right)$ $-F\left(x_{k}-0\right) \mid$; the left side of the first inequality on p. 657 should read $\left|\sum F\left(x_{k+1}-0\right)-F\left(x_{k}-0\right)\right|$.

$\ddagger$ Leçons sur l'Integration, Paris, 1928, pp. 296-307.

$\$$ T, p. 657, Theorem IX.

\| Loc. cit., p. 307.

I This transformation was suggested by S. Saks. It simplified essentially our discussion. 
tends to zero through positive values and through negative values respectively are the upper and lower right and left derived numbers of $F$ with respect to $\omega$, $D_{\omega} F^{+}, D_{\omega} F_{+} ; D_{\omega} F^{-}, D_{\omega} F_{-}$. This set of derived numbers we designate by $\Lambda_{\omega} F$. If these derived numbers are all equal then their common value is the derivative of $F$ with respect to $\omega, D_{\omega} F$. If $\omega$ is the variation function of $\alpha$ then $D_{\omega} \alpha=g= \pm 1$, except for at most a set of $\omega$-measure zero.* At points where $g= \pm 1$ we define the set $\Lambda_{\alpha} F$ by the relation $\Lambda_{\alpha} F=\Lambda_{\omega} F / g$. Where $g$ is different from \pm 1 the set $\Lambda_{\alpha} F$ is determined by considering the various limits as $h$ tends to zero of the ratio $\{F(x+h)-F(x)\} /\{\alpha(x+h)-\alpha(x)\}$. If the limit as $h$ tends to zero of $\psi(x, h)$ is equal to $a_{x}$ for $x+h$ taking on any values except those of a set of $\omega$-density zero $\dagger$ at $x$, then $a_{x}$ is the approximate derivative of $F$ with respect to $\omega, A D_{\omega} F$, and where $g= \pm 1, A D_{\alpha} F=A D_{\omega} F / g$.

2. The distribution of the values of the derived numbers of $F$ with respect to $\alpha$. Let $\omega$ be the variation function of $\alpha$, and $e_{c}$ the points of $(a, b)$ at which $\omega$ is continuous and which do not belong to intervals throughout which $\omega$ is constant. If $y=\omega(x)$, then according to our previous conventions $\ddagger$ the set $x_{i}$ of discontinuities of $\omega$ go into a countable set of open intervals $\beta_{i}=\left(b_{i}^{\prime}, b_{i}^{\prime \prime}\right)$ on the interval $\{\omega(a), \omega(b)\}=(\mu, \nu)$, and the countable set of intervals $\alpha_{j}$ throughout which $\omega$ is constant go into a countable set $y_{j}$ on $(\mu, \nu)$. Then, to each value of $y$ on this closed interval, except the set $y_{j}$ and the end points of $\beta_{i}$, there corresponds a single point $x_{y}$ on $(a, b)$. For such values of $y$ let $\phi(y)=F\left(x_{y}\right)$. At a point of the set $y_{j}$ let $\phi(y)$ have the constant value of $F$ on the corresponding interval of the set $\alpha_{j}$. At the end points $b_{i}^{\prime}, b_{i}^{\prime \prime}$ of the intervals $\beta_{i}$ let $\phi\left(b_{i}^{\prime}\right)=F\left(x_{i}-0\right)$, and $\phi\left(b_{i}^{\prime \prime}\right)=F\left(x_{i}+0\right)$.

The function $\phi(y)$ is now defined at every point of the closed interval $(\mu, \nu)$. Let $y_{c}$ be the set $\omega\left(e_{c}\right)$. At almost all points $y$ of $y_{c}$ the density of the set $\beta_{i}$ is zero. At such a point $y$ let us compare the various limits as $\Delta y$ tends to zero of the ratio

$$
\frac{\phi(y+\Delta y)-\phi(y)}{\Delta y},
$$

with the corresponding limits as $h$ tends to zero of the ratio

$$
\frac{F\left(x_{y}+h\right)-F(x)}{m \omega(x, h)} .
$$

* Daniell, these Transactions, vol. 19, p. 361. The result there given evidently holds under the present definition of a derivative.

$\dagger$ T, p. 662, where right hand $\omega$-density of the set $E$ is defined. $\omega$-density is the limit as $h$ tends to zero of the ratio $\bar{m} E(x-h, x+h) / m \omega(x-h, x+h)$.

$\ddagger T$, p. 646, 1. 
If $y+\Delta y$ is a point of $y_{c}$ and $x_{v}+h$ is the corresponding point of $e_{c}$, then the two ratios are the same. This is also the case if $y+\Delta y$ is a point of $y_{j}$, and $x_{y}+h$ is on the corresponding interval $\alpha_{j}$. Let $y+\Delta y$ be a point of $\beta_{i}$. Then if $x_{y}+h=x_{i}$ we have

$$
F\left(x_{y}+h\right)=\phi(y+\Delta y), m \omega(x, h)=\Delta y+t_{i},
$$

where $\left|t_{i}\right| \leqq m \beta_{i}$. Consider the ratio

$$
\frac{m \omega(x, h)}{\Delta y}=\frac{\Delta y+t_{i}}{\Delta y}=1+\frac{t_{i}}{\Delta y} .
$$

Now

$$
\left|\frac{t_{i}}{\Delta y}\right| \leqq \frac{m \beta_{i}}{|\Delta y|} .
$$

And since at the point $y$ the density of the set of intervals $\beta_{i}$ is zero, it follows that

$$
\frac{m \beta_{i}}{|\Delta y|+\left|t_{i}\right|}=\frac{1}{\frac{|\Delta y|}{m \beta_{i}}+\frac{\left|t_{i}\right|}{m \beta_{i}}}
$$

tends to zero with $\Delta y$. As a result of this, and the fact that $\left|t_{i}\right| / m \beta_{i} \leqq 1$, we conclude that $m \beta_{i} /|\Delta y|$ tends to zero with $\Delta y$. Relations (4) and (5) then show that

$$
\lim _{\Delta y \rightarrow 0} \frac{m \omega(x, h)}{\Delta y}=1 .
$$

It then follows from (3) and (6) that if $y+\Delta y$ is a point of $\beta_{i}$, and $x_{y}+h=x_{i}$, where $x_{i}$ is the point of discontinuity of $\omega$ corresponding to the interval $\beta_{i}$, then the ratios (1) and (2) have the same limits of indetermination.

It remains to consider the case in which $y+\Delta y$ is an end point of $\beta_{i}$. Let $y+\Delta y=b_{i}^{\prime}$. Then $\phi(y+\Delta y)=\phi\left(b_{i}^{\prime}\right)=F\left(x_{i}-0\right)$. Let $x_{l}$ be a sequence of values of $x$ belonging to $e_{c}$ or to $\alpha_{j}$, and tending to $x_{i}$ from the left. Then $F\left(x_{l}\right)$ tends to $F\left(x_{i}-0\right)=\phi(y+\Delta y)$, and if $x_{y}+h=x_{l}$ then $m \omega\left(x_{y}, h\right)$ tends to $\Delta y$. Thus for $l$ sufficiently large the ratio (2) is arbitrarily near to the ratio (1). A like manner of reasoning may be used to show that the same situation prevails when $y+\Delta y=b_{i}^{\prime \prime}$.

We have now proved that every value that is approached by the ratio (1) as $\Delta y$ tends to zero, is also approached by the ratio (2) as $h$ tends to zero over a suitably chosen sequence of values of $h$. Starting with the ratio (2) and 
letting $h$ tend to zero through all possible values, it can be shown by reasoning similar to the above that for every limit approached by (2) there is a sequence of values of $\Delta y$ tending to zero over which the ratio (1) approaches the same limit. It then follows that, except for a part of $e_{c}$ of $\omega$-measure zero, the distribution of the values of the set $\Lambda_{\omega} F$ at the points of $e_{c}$ is the same as the distribution of the values of the derived numbers of $\phi(y)$ at the points of $y_{c}$. At the set $x_{i}$ of discontinuities of $\omega, D_{\omega} F$ exists and is finite. If $\omega$ is the variation function of $\alpha$, then where $D_{\omega} \alpha=g= \pm 1, \Lambda_{\alpha} F=\Lambda_{\omega} F / g$. This relation holds except for a set of $\omega$-measure zero. At a point where $g=-1$ an upper derived number with respect to $\omega$ may correspond to a lower derived number with respect to $\alpha$, and conversely. But where one is finite the other is also. Furthermore, if the function $F$ is measurable relative to $\alpha^{*}$ on $(a, b)$, then the function $\phi$ is measurable on $(\mu, \nu)$. Consequently, if we take into consideration the known facts concerning the distribution of the values of the derived numbers of measurable functions $\dagger$ we have the following result:

Let $\alpha(x)$ be a function of bounded variation on the interval $(a, b)$, and let the function $F(x)$ be finite at each point of $(a, b)$, measurable relative to $\alpha$ on $(a, b)$, constant on intervals throughout which $\alpha$ is constant, and such that at the points of discontinuity of $\alpha, F(x-0)$ and $F(x+0)$ both exist. Then, except for at most a set of $\alpha$-measure zero, the derived numbers and approximate derivatives of $F$ with respect to $\alpha$ fall into one or the other of the following classes:

$$
A D_{\alpha} F \text { exists and is finite. }
$$

The points of class (1) are of four types:

$$
\begin{array}{cl}
D_{\alpha} F \text { exists and is finite. } & \\
D_{\alpha} F_{+}=A D_{\alpha} F=D_{\alpha} F^{-}, & D_{\alpha} F^{+}=\infty, D_{\alpha} F_{-}=-\infty . \\
D_{\alpha} F^{+}=A D_{\alpha} F=D_{\alpha} F_{-}, & D_{\alpha} F_{+}=-\infty, D_{\alpha} F^{-}=\infty . \\
D_{\alpha} F^{+}=D_{\alpha} F^{-}=\infty, & D_{\alpha} F_{+}=D_{\alpha} F_{-}=-\infty .
\end{array}
$$

3. The determination of $F(x)$ by means of the derived numbers of $F$ with respect to $\alpha$. In this section, in addition to the conditions imposed above, $F$ is continuous where $\alpha$ is continuous, and, at points of discontinuity of $\alpha, F(x)$ lies on the interval defined by $F(x-0)$ and $F(x+0)$. Furthermore, the region of definition of $F$ is extended beyond the interval $(a, b)$ in such a way that

* T, p. 646, §1, p. $655, \S 6$.

$\dagger$ J. C. Burkill and U.S. Haslam-Jones, The derivates and approximate derivates of measurable functions, Proceedings of the London Mathematical Society, (2), vol. 32, pp. 346-355. 
$F(a-0)=F(a)$, and $F(b+0)=F(b)$. Let $\omega$ be the variation function of $\alpha$. On the interval $\omega(a)=\mu \leqq y \leqq \nu=\omega(b)$ let $\psi(y)$ be the function $\phi$ defined in the previous section, except for the intervals $\beta_{i}$, where $\psi$ is linear, ranging from $F\left(x_{i}-0\right)$ to $F\left(x_{i}\right)$ on the left half of $\beta_{i}$ and from $F\left(x_{i}\right)$ to $F\left(x_{i}+0\right)$ on the right half of this interval. We prove the following:

If $D_{\omega} F^{+}$is finite at each point of $(a, b)$, then $D \psi^{+}$is finite at each point of $(\mu, \nu)$, with the possible exception of the right hand end points of the intervals $\beta_{i}$.

On the intervals $\beta_{i}$ the function $\psi$ is linear, and consequently $D \psi^{+}$is finite at each point $y$ for which $b_{i}^{\prime} \leqq y<b_{i}^{\prime \prime}$. For $y$ a point of $y_{c}$ and $y+\Delta y$ a point of $y_{c}$ or $y_{i}$, the limits of indetermination of the ratios

$$
\frac{\psi(y+\Delta y)-\psi(y)}{\Delta y} \text { and } \frac{F\left(x_{y}+h\right)-F\left(x_{y}\right)}{m \omega\left(x_{y}, h\right)}
$$

are the same provided $x_{y}+h$ is so chosen that $y+\Delta y=\omega\left(x_{y}+h\right)$. The same statement holds if $y$ is a point of $y_{j}$, provided $x_{y}$ is the right hand end point of $\alpha_{j}$, and $F\left(x_{y}\right)$ is replaced by $F\left(x_{y}-0\right)$. Hence, if $D_{\omega} F^{+}$is finite, either the upper limit of the first ratio is finite or this ratio becomes positively infinite as $\Delta y$ tends to zero with $y+\Delta y$ on intervals of the set $\beta_{i}$. Let $y+\Delta y$ be on $\beta_{i}=\left(b_{i}^{\prime}, b_{i}^{\prime \prime}\right)$; let $b_{i}^{\prime}-y=\Delta^{\prime} y, \Delta y=\Delta^{\prime} y+t_{i}^{\prime}$, and let $m \beta_{i}=t_{i}$. There are then two cases to consider: (i) $\Delta^{\prime} y / t_{i}$ bounded from zero; (ii) $\Delta^{\prime} y / t_{i}$ tending to zero as $\Delta^{\prime} y$ tends to zero. In case (i) the ratio $\Delta \psi / \Delta y$ lies between the two ratios

i.e., between

$$
\frac{F\left(x_{i}+0\right)-F\left(x_{y}\right)}{\Delta^{\prime} y+t_{i}^{\prime}} \text { and } \frac{F\left(x_{i}-0\right)-F\left(x_{y}\right)}{\Delta^{\prime} y+t_{i}^{\prime}},
$$

$$
\frac{F\left(x_{i}+0\right)-F\left(x_{y}\right)}{\Delta^{\prime} y+t_{i}} / \frac{\Delta^{\prime} y+t_{i}^{\prime}}{\Delta^{\prime} y+t_{i}} \text { and } \frac{F\left(x_{i}-0\right)-F\left(x_{y}\right)}{\Delta^{\prime} y} / \frac{\Delta^{\prime} y+t_{i}^{\prime}}{\Delta^{\prime} y} .
$$

Since $\Delta^{\prime} y+t_{i}=m \omega\left(x_{y}, x_{i}\right), \Delta^{\prime} y=m \omega\left(x_{y}, x_{i}-0\right)$, and since $D_{\omega} F^{+}$is finite, it follows that the numerators of these last two expressions are bounded above, and since $\Delta^{\prime} y / t_{i}$ is bounded from zero, it follows that their denominators are bounded from zero. Thus it has been shown that $D \psi^{+}<\infty$. It remains to be shown that $D \psi^{+}>-\infty$. If the ratio $\Delta \psi / \Delta y$ becomes negatively infinite for every sequence of values of $\Delta y$, then the ratio $\Delta F / \Delta \omega$ becomes negatively infinite for $x_{\nu}+h$ points of $e_{c}$ or $\alpha_{j}$. Hence, since $D_{\omega} F^{+}$is finite, we must have $\Delta F / \Delta \omega$ tending to a finite limit for $x_{y}+h$ points of the set $x_{i}$ of discontinuities of $\omega$. In this case,

$$
\frac{\Delta F}{m \omega\left(x_{y}, h\right)}=\frac{F\left(x_{i}\right)-F\left(x_{y}\right)}{m \omega\left(x_{y}, x_{i}\right)}=\frac{\psi(y+\Delta y)-\psi(y)}{m \omega\left(x_{y}, x_{i}\right)},
$$


where $\Delta y=\Delta^{\prime} y+t_{i} / 2$ and $m \omega\left(x_{y}, x_{i}\right)=\Delta^{\prime} y+t_{i}$. But this makes $\Delta y / m \omega\left(x_{y}, x_{i}\right)$ $\geqq \frac{1}{2}$. Hence, if $\Delta \psi / \Delta y$ becomes negatively infinite, so does $\Delta \psi / \Delta \omega$ and its equivalent ratio $\Delta F / m \omega\left(x_{y}, x_{i}\right)$, from which it follows that $D_{\omega} F^{+}=-\infty$. But this is a contradiction. We can, therefore, conclude that when $\Delta^{\prime} y / t_{i}$ is bounded from zero, $D \psi^{+}$is finite.

Remark. In case (i) if the function $\psi$ is defined as above, except that it is a single linear function on $\beta_{i}$ ranging from $F\left(x_{i}-0\right)$ to $F\left(x_{i}+0\right)$, the same conclusions hold in regard to $D \psi^{+}$. It was this definition of $\psi$ that was suggested by Saks. But if $\psi$ is defined in this manner there exist functions $F$ and $\omega$ such that in case (ii) $D_{\omega} F^{+}$is finite and $D \psi^{+}=-\infty$. We exhibit such an example. It throws light on the whole situation.

On the interval $(0, e)$ let

$$
x_{n}=\sum_{i=n}^{\infty} \frac{1}{i !}, \text { and let } \omega(x)=\sum_{i=n}^{\infty} \frac{1}{i !} \text { on } x_{n} \leqq x<x_{n-1} .
$$

It is easily verified that if $\beta_{n}=\omega\left(x_{n}-0\right)<y<\omega\left(x_{n}+0\right)$ then for $y=0, \Delta^{\prime} y / t_{i}$ tends to zero as $\Delta^{\prime} y$ tends to zero, which is the condition of case (ii). Let $F(0)=0$, and on $x_{n}<x \leqq x_{n-1}$ let

$$
F(x)=-\sum_{i=n-1}^{\infty} \frac{1}{i !} ; \text { then } \frac{F\left(x_{n-1}\right)-F(0)}{m \omega\left(0, x_{n-1}\right)}=-1,
$$

which shows that $D_{\omega} F^{+}$is finite. Also

$$
\frac{F\left(x_{n-1}+h\right)-F(0)}{m \omega\left(0, x_{n-1}+h\right)}=\frac{-\sum_{i=n-2}^{\infty} \frac{1}{i !}}{\sum_{i=n-1}^{\infty} \frac{1}{i !}}=-(n-1) \frac{1+\frac{1}{n-1}+\frac{1}{n(n-1)}+\cdots}{1+\frac{1}{n}+\frac{1}{n(n+1)}+\cdots},
$$

which becomes negatively infinite as $n$ increases. Hence $D_{\omega} F_{+}=-\infty$. Now let $\psi$ be linear on $\beta_{n}$ and range from $F\left(x_{n}-0\right)$ to $F\left(x_{n}+0\right)$. Then if $y=0$, and $y+\Delta y$ is on $\beta_{n}$,

$$
\frac{\Delta \psi}{\Delta y}=\frac{\sum_{i=n-1}^{\infty} \frac{1}{i !}-t_{i}^{\prime \prime}}{\sum_{i=n}^{\infty} \frac{1}{i !}+t_{i}^{\prime}}=-n \frac{\theta_{1}(n)+n(n-1) ! t_{i}^{\prime}}{\theta_{2}(n)+n ! t_{i}^{\prime}}
$$

since $t_{i}^{\prime \prime} / t_{i}^{\prime}=n$. The functions $\theta_{1}(n)$ and $\theta_{2}(n)$ tend to unity as $n$ increases. From this it follows that the last member of the foregoing equality, and consequently $\Delta \psi / \Delta y$, becomes negatively infinite as $n$ increases. Hence $D \psi^{+}=-\infty$. 
We now show that for the function $\psi$ defined at the beginning of this section we have in case (ii), just as in case (i), that $D_{\omega} F^{+}$finite implies $D \psi^{+}$finite. If $D_{\omega} F^{+}$is finite and $D \psi^{+}=+\infty$, then $\Delta \psi / \Delta y$ must become infinite for $y+\Delta y$ on $\beta_{i}$. We then have

$$
\begin{aligned}
\frac{\Delta \psi}{\Delta y}= & \frac{\psi(y+\Delta y)-\psi\left(y+\Delta^{\prime} y\right)}{t_{i}^{\prime}} / \frac{\Delta^{\prime} y+t_{i}^{\prime}}{t_{i}^{\prime}} \\
+ & \frac{\psi\left(y+\Delta^{\prime} y\right)-\psi(y)}{\Delta^{\prime} y} / \frac{\Delta^{\prime} y+t_{i}^{\prime}}{\Delta^{\prime} y}
\end{aligned}
$$

The denominators of both ratios on the right are greater than or equal to unity, and since $D_{\omega} F^{+}$is finite the numerator of the second ratio is finite. Hence if $\Delta \psi / \Delta y$ becomes positively infinite so does the numerator of the first ratio. If we set $\psi(y+\Delta y)-\psi\left(y+\Delta^{\prime} y\right)=t_{i}^{\prime \prime}$, then $t_{i}^{\prime \prime} / t_{i}^{\prime}$ becomes infinite, and

$$
\frac{\Delta \psi}{\Delta y}=\frac{F\left(x_{i}-0\right)-F\left(x_{y}\right)}{\Delta^{\prime} y+t_{i}^{\prime}}+\frac{t_{i}^{\prime \prime}}{\Delta^{\prime} y+t_{i}^{\prime}}=A+B .
$$

Let us compare $A$ and $B$ with

$$
A^{\prime}=\frac{F\left(x_{i}-0\right)-F\left(x_{y}\right)}{\Delta^{\prime} y+\tau_{i}^{\prime}} \text { and } B^{\prime}=\frac{\tau_{i}^{\prime \prime}}{\Delta^{\prime} y+\tau_{i}^{\prime}}
$$

where $t_{i}^{\prime \prime} \leqq \tau_{i}^{\prime \prime} \leqq F\left(x_{i}\right)$, and $t_{i}^{\prime} \leqq \tau_{i}^{\prime} \leqq t_{i} / 2$. If $A$ is negative then $A^{\prime} \geqq A$, and if $A$ is positive, $A^{\prime} \geqq 0$. Since $t_{i}^{\prime \prime} / t_{i}^{\prime}=\tau_{i}^{\prime \prime} / \tau_{i}^{\prime}$ it follows that $B^{\prime} \geqq B$. Since $\Delta^{\prime} y / t_{i}$ tends to zero, and since $\tau_{i}^{\prime \prime} / \tau_{i}^{\prime}$ becomes positively infinite, it follows that $B^{\prime}$ becomes infinite as $\Delta^{\prime} y$ tends to zero and $\tau_{i}^{\prime}$ tends to $t_{i} / 2$. Hence, if $\Delta \psi / \Delta y$ becomes positively infinite, so does $A^{\prime}+B^{\prime}$ for $\tau_{i}^{\prime}=t_{i} / 2$. But this means that the ratio $\left\{F\left(x_{i}\right)-F\left(x_{y}\right)\right\} / t_{i}$ tends to $+\infty$. Then, since $\Delta^{\prime} y / t_{i}$ tends to zero, it follows that $\left\{F\left(x_{i}\right)-F\left(x_{y}\right)\right\} / m \omega\left(x_{y}, x_{i}\right)$ tends to $+\infty$. But this means that, at the point $x_{y}, D_{\omega} F^{+}=+\infty$, which is a contradiction. We conclude, therefore, that $D \psi^{+}<\infty$. The proof that $D \psi^{+}>-\infty$ is the same as in case (i).

We now know that if, at each point of $(a, b), D_{\omega} F+$ is finite, then, at each point of $(\mu, \nu), D \psi^{+}$is finite, except possibly the right hand end points of the intervals $\beta_{i}$, at which points the left hand derivative of $\psi$ exists and is finite. Hence if, at each point of $(a, b), D_{\omega} F^{+}$is finite, then at each point of $(\mu, \nu)$ one of the derived numbers of $\psi$ is finite. At the points of $y_{c}$, except at most a null set, this finite derived number can be taken as $D \psi^{+}$, and will be equal to $D_{\omega} F^{+}$at the corresponding points of $e_{c}$, which is all of $e_{c}$ except at most a set of $\omega$-measure zero. Furthermore, on an interval of the set $\beta_{i}$, 


$$
\int_{b_{i}^{\prime}}^{b_{i}^{\prime \prime}} D \psi^{\prime} d y=\psi\left(b_{i}^{\prime \prime}\right)-\psi\left(b_{i}^{\prime}\right)=F\left(x_{i}+0\right)-F\left(x_{i}-0\right)=\int_{x_{i}} D_{\omega} F d \omega .
$$

Let $a^{\prime} \leqq x \leqq a^{\prime \prime}$ be an interval on $(a, b)$ with $\left(a^{\prime}, a^{\prime \prime}\right)$ points of continuity of $\omega$, and $b^{\prime}=\omega\left(a^{\prime}\right) \leqq y \leqq b_{i}^{\prime \prime}=\omega\left(a^{\prime \prime}\right)$, the corresponding interval on $(\mu, \nu)$. Let $f(y)=D \psi^{+}$where $D \psi^{+}$is finite, and otherwise let $f(y)$ be the left hand derivative of $\psi$. Then

$$
\int_{b^{\prime}}^{b^{\prime \prime}} f(y) d y=\psi\left(b^{\prime \prime}\right)-\psi\left(b^{\prime}\right)=F\left(a^{\prime \prime}\right)-F\left(a^{\prime}\right),
$$

where the integration is in the sense of Denjoy.* Since at almost all of $y_{c}$ the function $f(y)=D_{\omega} F^{+}$at the corresponding points of $e_{c}$, and since the integral of $f(y)$ over $\beta_{i}$ is equal to the integral of $D_{\omega} F$ over $x_{i}$, it follows that $D_{\omega} F^{+}$is Denjoy integrable with respect to $\omega \dagger$ on $\left(a^{\prime}, a^{\prime \prime}\right)$, and that

$$
\int_{a^{\prime}}^{a^{\prime \prime}} D_{\omega} F^{+} d \omega=\int_{b^{\prime}}^{b^{\prime \prime}} f(y) d y=F\left(a^{\prime \prime}\right)-F\left(a^{\prime}\right) .
$$

Now let $(l, m)$ be any interval on $(a, b),\left(a_{n}^{\prime}, a_{n}^{\prime \prime}\right)$ a sequence of intervals for which $l<\cdots<a_{2}^{\prime}<a_{1}^{\prime}<a_{1}^{\prime \prime}<a_{2}^{\prime \prime}<\cdots<m$, where $a_{n}^{\prime}$ and $a_{n}^{\prime \prime}$ are points of continuity of $\omega, a_{n}^{\prime}$ tending to $l$ and $a_{n}^{\prime \prime}$ tending to $m$. Then

$$
F(m-0)-F(l+0)=\lim _{n \rightarrow \infty} \int_{a_{n^{\prime}}}^{a_{n^{\prime \prime}}} D_{\omega} F^{+} d \omega=\int_{l<x<m} D_{\omega} F^{+} d \omega,
$$

where the integration with respect to $\omega$ is in the sense of Denjoy. Also

$$
\begin{aligned}
F(l+0)-F(l-0) & =\int_{l} D_{\omega} F^{+} d \omega, \\
F(m+0)-F(m-0) & =\int_{m} D_{\omega} F^{+} d \omega .
\end{aligned}
$$

By putting $l=a$ and $x=m$ these results then permit us to state the following theorem:

If $f(x)$ is finite at each point of $a \leqq x \leqq b$, and is the upper right derivative with respect to a non-decreasing function $\omega$, where $F$ satisfies the conditions laid 'down at the beginning of this section, then if $x$ is any point on $(a, b)$,

* Lebesgue, loc. cit., p. 150.

† T, p. 665, \$10. 


$$
F(x-0)-F(a)=\int_{a \leq t<x} f(t) d \omega, F(x+0)-F(a)=\int_{a \leq t \leq x} f(t) d \omega,
$$

where the integration with respect to $\omega$ is in the sense of Denjoy.

In a similar manner the same result can be established for any of the other derived numbers of $F$ with respect to $\omega$. If it is known that $f$ is equal to one of the derived numbers of $F$ with respect to $\omega$, not necessarily the same derived number at each point, and if further for the intervals $\alpha_{i}$ throughout which $\omega$ is constant $f$ is a right hand derived number at the upper end, or a left hand derived number at the lower end, then it follows by reasoning similar to the above that one of the derived numbers of $\psi$ is finite at each point of $(\mu, \nu)$, and this in turn leads to the truth of the foregoing theorem in the present case.

If $\alpha$ is a function of bounded variation on $(a, b)$ and $\omega$ the variation function of $\alpha$, then where $g= \pm 1=D_{\omega} \alpha, \Lambda_{\alpha} F=\Lambda_{\omega} F / g$. Hence, if at such points one of the set $\Lambda_{\alpha} F$ is finite, then one of the set $\Lambda_{\omega} F$ is finite. Where $g$ is different from \pm 1 , the set $\Lambda_{\alpha} F$ is determined from the ratio $\Delta F / \Delta \alpha$. It is not difficult to construct functions $F$ and $\alpha$ for which $D_{\alpha} F^{+}$is finite, $D_{\omega} F^{+}=+\infty$, $D_{\omega} F_{+}=-\infty$. If, however, at this exceptional set for all $|h|$ sufficiently small, $\Delta \alpha=\alpha(x+h)-\alpha(x)$ does not change sign unless $h$ changes sign, it is easily shown that one of the set $\Lambda_{\alpha} F$ finite implies one of the set $\Lambda_{\omega} F$ finite. When the function $\alpha$ satisfies this condition, we have

If the function $f(x)$ is finite at each point of the interval $a \leqq x \leqq b$ and equal to one of the derived numbers of $F$ with respect to $\alpha$, for the intervals of the set $\alpha_{j}$ throughout which $\alpha$ is constant either a right hand derived number at the upper end or a left hand derived number at the lower end, then

$$
F(x-0)-F(a)=\int_{a \leq t<x} f(t) d \alpha, F(x+0)-F(a)=\int_{a \leq t \leq x} f(t) d \alpha,
$$

where the integration with respect to $\alpha$ is in the sense of Denjoy.*

At the points for which $g= \pm 1, f=\theta / g$ where $\theta$ is one of the derived numbers of $F$ with respect to $\omega$. If we set $\theta_{1}(x)=\theta(x)$ where $g= \pm 1$, and $\theta_{1}$ equal to a finite derived number of $F$ with respect to $\omega$ at the remaining points of $(a, b)$, then on the intervals $a \leqq t<x$ and $a \leqq t \leqq x$, the function $\theta_{1}$ is integrable in the sense of Denjoy with respect to $\omega$ to the values $F(x-0)-F(a)$ and

* The integral of $f d \alpha$ is defined as the integral of $f g d \omega$, in whatever sense the latter integral exists, T, p. 655, §6. It is stated, T, p. 675, $\S 13$, that if the integral of $f d \omega$ exists in the sense of Denjoy then the integral of $f g d \omega$ exists in the same sense. Obviously, this is only necessarily true when $f$ is summable with respect to $\omega$, since $g$ may be negative where $f$ is negative and positive where $f$ is positive. 
$F(x+0)-F(a)$ respectively. Hence for the interval $(a, x)$ we have, since $\theta=\theta_{1}$ except for at most a set of $\omega$-measure zero,

$$
\int f d \alpha=\int f g d \omega=\int \theta d \omega=F(x \mp 0)-F(a),
$$

Fholding according as the integration is taken over the interval $a \leqq t<x$, or $a \leqq t \leqq x$. This establishes the theorem.

Acadia University,

Wolfville, Nova Scotia 